\title{
LONGITUDINAL CONTROL OF A FIXED WING UAV
}

\author{
David Villota Miranda, Montserrat Gil-Martínez, Javier Rico-Azagra \\ Control Engineering Group, Electrical Engineering Department, University of La Rioja \\ david.villota@alum.unirioja.es, \{montse.gil, javier.rico\}@unirioja.es
}

\begin{abstract}
Summary
This article presents an approach to the mathematical model of a fixed wing unmanned aerial vehicle prototype. The model is split in two different parts, related to the longitudinal and lateral stability, respectively. For this, Newton-Euler formulation is used as well as basic aerodynamic theory. Aerodynamic coefficients, inertias and characteristic points of the aircraft are obtained through simulations with an open-source software called XFLR-5, and the physical parameters of the model match the prototype's. Then, a longitudinal control strategy describes the altitude control in a cascade architecture, whose inner loop conveniently manoeuvres the pitch angle by acting on the symmetric flag deflection. Frequency domain techniques are used to design PID controllers.
\end{abstract}

Key words: Fixed-wing unmanned aerial vehicle (UAV), stability derivatives, cascade control, proportional integral derivative control.

\section{Introduction}

The unmanned aerial vehicles (UAV) are becoming more and more popular as they can be used in a wide range of fields. The technology involved is being continuously developed and its price is constantly decreasing [1]. Every time open-source projects are being carried out by research groups and more and more information can be found on the internet, given by Radio Control fan communities.

In the UAV field, of the four different categories, it is micro and mini that have experimented a greater expansion. Even though multirotors are the most popular, glider UAVs have more advantages as their autonomy is greater. That is why they are used in field recognition or to reach further places that multi-rotors cannot [2].

The fixed wing architecture has been chosen as it is easier to maintain than a conventional plane, and more resistant in case of crushing. Normally the tail suffer the worst part, as the connection to the main body is weak.

Regarding control, it is challenging since, in contrast to the conventional architecture, where there are multiple control surfaces, in a fixed wing aircraft we can only control the flap deflection in a symmetric or asymmetric way, depending which stability (longitudinal or lateral) is to be controlled.

For this work, an unconventional plane (fixed wing architecture) has been built with all the required avionics and actuators (servo motors, Ardupilot, ESC,...). Figure 1 illustrates its dimensional characteristics. The final aim is the development of a navigation and control system not to depend on commercial solutions. As a preliminary work, this paper describes the mathematical modelling of the fixed wing prototype, and focuses on the linear model for longitudinal stability. Newton-Euler formulation [3] is being used, but the mayor difficulty is the identification of aerodynamically coefficients and characteristics points that will be obtained through simulations with an open-source software called XFLR-5 [4]. Then, a control strategy is being presented in order to firstly control the pitch angle, which modifies the angle of attack that modifies the magnitude of the lift force. This ultimately leads to proper altitude control. The system to be controlled is multivariable, non-linear and highly coupled.

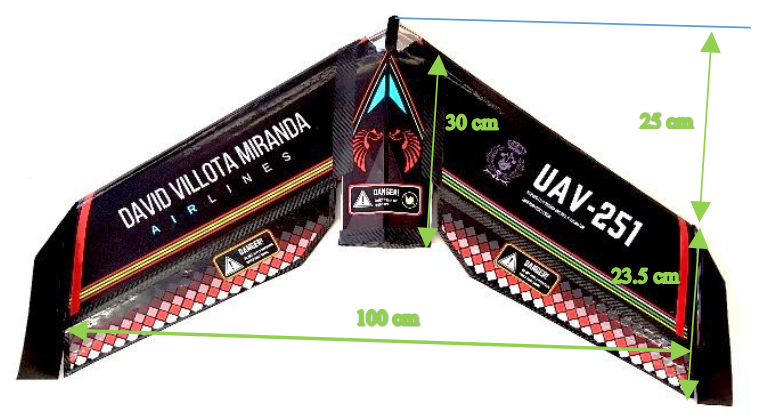

Figure 1: Aircraft prototype

\section{2. $\quad$ Fixed wing UAV modelling}

UAV movement is defined by 6 degrees of freedom (DoF) in the earth inertial frame (E): 3 coordinates for spatial positioning $[x, y, z]^{\mathrm{T}}$ and 3 angles for orientation 
$[\phi, \theta, \psi]^{\mathrm{T}}$. A fixed wing is an unconventional type of aircraft with only two control surfaces (flaps) for manoeuvring (Figure 2). The symmetric deflection ( $\delta_{E}$ ) of flaps will act in the control of the longitudinal motion variables $(\theta, z)$, and the asymmetric deflection $\left(\delta_{A}\right)$ of flaps will act in the control of the lateral motion variables $(\phi, \psi)$. The controlled attitude $(\theta, \phi)$, and heading $(\psi)$ are further responsible of spatial displacement.
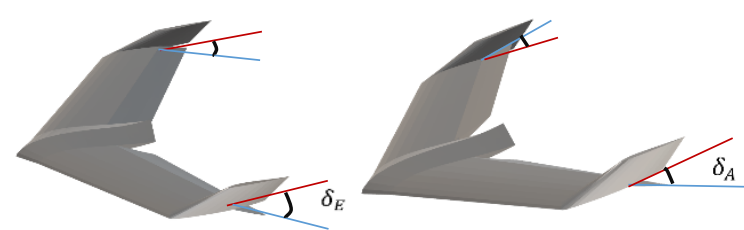

Figure 2: Symmetric $\delta_{E}$ and asymmetric $\delta_{A}$ deflections

Linear $[u, v, w]^{T}$ and angular $[p, q, r]^{T}$ velocities of the fixed wing are measured in the body frame (B). Figure 3 depicts the three orthogonal axis of this second reference frame, which is clamped to the mass centre of the vehicle.

In all aerodynamic systems, special attention should be payed to the "wind frame" (W), whose X-axis is parallel to the air velocity vector $V_{a}$. W reference frame involves a rotation $\alpha$ (attack angle) with respect to the body $\mathrm{Y}$-axis and a rotation $\beta$ (sweep angle) with respect to the body Z-axis, as Figure 3 illustrates. $V_{a}$ magnitude depends on the relative vehicle's forward airspeed.

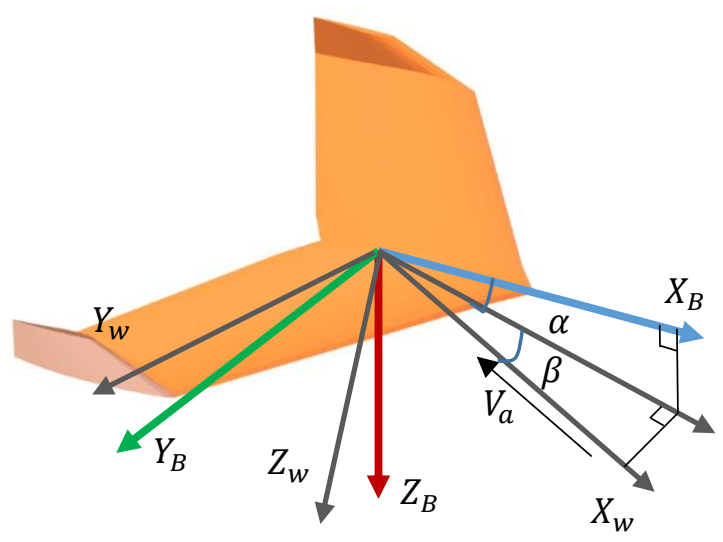

Figure 3: Body clamped frame (colour) and wind frame (grey)

\subsection{Equations of motion in the body frame}

Newton's second law on the linear momentum yields the contribution of the three force components in the body frame:

$$
\left\{\begin{array}{l}
F_{x}=m(\dot{u}+q w-r v) \\
F_{y}=m(\dot{v}+r u-w q) \\
F_{z}=m(\dot{w}+p v-u q)
\end{array}\right.
$$

where $m$ is the plane mass.

Euler's second law on the angular momentum yields the contribution of the three torque components in the body frame:

$$
\left\{\begin{array}{l}
M_{x}=I_{x x} \dot{P}+\left(I_{x x}-I_{y y}\right) Q R+I_{x z} \dot{P}+I_{x z} P Q \\
M_{y}=I_{y y} \dot{Q}+\left(I_{x x}-I_{z z}\right) P R+\left(R^{2}+P^{2}\right) I_{x z} \\
M_{z}=I_{z z} \dot{R}+\left(I_{y y}-I_{x x}\right) P Q+I_{x z} \dot{P}-I_{x z} Q R
\end{array}\right.
$$

being the inertial tensor:

$$
I=\left[\begin{array}{ccc}
I_{x x} & 0 & I_{x z} \\
0 & I_{y y} & 0 \\
I_{z x} & 0 & I_{z z}
\end{array}\right]
$$

Mass and inertial moments for the fixed wing prototype in this work are in Table 1, together with other relevant parameters. Moments of inertia have been computed using the $3 \mathrm{D}$ simulation program XFLR-5 [4].

\begin{tabular}{|l|c|c|}
\hline Parameters & Values & Units \\
\hline$m$ & 0.9 & $\mathrm{~kg}$ \\
\hline$A$ & 0.27 & $\mathrm{~m}^{2}$ \\
\hline$b$ & 1 & $\mathrm{~m}$ \\
\hline$c$ & 0.27 & $\mathrm{~m}$ \\
\hline$V_{a}$ & 31 & $\mathrm{~m} / \mathrm{s}$ \\
\hline$\alpha$ & -0.5 & $\underline{\mathbf{o}}$ \\
\hline$\beta$ & 0 & $\underline{\mathbf{o}}$ \\
\hline$g$ & -9.81 & $\mathrm{~m} / \mathrm{s}^{2}$ \\
\hline$k_{d}$ & $8.5 * 10^{-9}$ & $\mathrm{~N} \cdot \mathrm{m} \cdot \mathrm{s}^{2} / \mathrm{rad}$ \\
\hline$k_{t}$ & $5.65 * 10^{-7}$ & $\mathrm{~N} \cdot \mathrm{m} \cdot \mathrm{s} / \mathrm{rad}$ \\
\hline$I_{x x}$ & 0.02381 & $\mathrm{~kg} \cdot \mathrm{m}^{2}$ \\
\hline$I_{y y}$ & 0.00841 & $\mathrm{~kg} \cdot \mathrm{m}^{2}$ \\
\hline$I_{z z}$ & 0.03222 & $\mathrm{~kg} \cdot \mathrm{m}^{2}$ \\
\hline$I_{x z}=-I_{z x}$ & 0 & $\mathrm{~kg} \cdot \mathrm{m}^{2}$ \\
\hline $\boldsymbol{I}_{\boldsymbol{j}}^{X}$ & $2.44 * 10^{-6}$ & $\mathrm{~kg} \cdot \mathrm{m}^{2}$ \\
\hline
\end{tabular}

Table 1. Parameters of fixed wing prototype and flight conditions

\subsection{External strengths and torques}

The relative vehicle's forward airspeed $V_{a}$ exerts an aerodynamic strength due to the variance of pressure

$$
Q=\frac{1}{2} \rho V_{a}^{2} A
$$


between the upper and lower parts of the wing, whose surface is $A ; \rho$ is the air density. Thus, the drift $(D)$, sweep $(S)$ and lift $(L)$ components of the aerodynamic strength in the wind frame are

$$
\mathbf{F}_{a}^{\mathrm{W}}=\left[\begin{array}{c}
-D \\
S \\
-L
\end{array}\right]=\frac{1}{2} \rho V_{a}^{2} A\left[\begin{array}{c}
-C_{D} \\
C_{S} \\
-C_{L}
\end{array}\right]
$$

being $C_{D}, C_{S}$, and $C_{L}$ the aerodynamic coefficients in each $\mathrm{W}$ axis. Then, the rotation matrix

$$
\mathbf{R}^{\mathrm{B} \leftarrow \mathrm{W}}=\left(\begin{array}{ccc}
\cos \alpha \cos \beta & -\cos \alpha \sin \beta & -\sin \beta \\
\sin \beta & \cos \beta & 0 \\
\sin \alpha \cos \beta & -\sin \alpha \cos \beta & \cos \alpha
\end{array}\right)
$$

is applied to obtain those strengths in the body frame:

$$
\mathbf{F}_{a}=\mathbf{R}^{\mathrm{B} \leftarrow \mathrm{W}} \mathbf{F}_{a}^{\mathrm{W}}=\left[\begin{array}{l}
F_{a_{X}} \\
F_{a_{Y}} \\
F_{a_{Z}}
\end{array}\right]
$$

The application point of $\mathbf{F}_{a}$ can slightly change depending on the attack $\alpha$ and sweep $\beta$ angles. In order to simplify the problem, the application point is considered fixed and roll $(L)$, pitch $(M)$ and yaw $(N)$ moments

$$
\mathbf{M}_{\boldsymbol{a}}=\left[\begin{array}{c}
L \\
M \\
N
\end{array}\right]=\frac{1}{2} \rho V_{a}^{2} A\left[\begin{array}{c}
-b C_{l} \\
c C_{m} \\
-b C_{n}
\end{array}\right]
$$

are added to correct this assumption; $b$ and $c$ are the wing span and chord, respectively; Table 1 details their values for this work prototype. $C_{l}, C_{m}, C_{n}$ are aerodynamic coefficients in each axis. They depend on the attack angle $(\alpha)$, the flap deflection $\left(\delta_{E}, \delta_{A}\right)$ and the angular velocities $[p, q, r]^{T}$. Translational (5) and rotational (8) aerodynamic coefficients have been calculated following the equations in [3].

A tail propeller rotates at $\omega_{j}$, which provides a thrust force along the $\mathrm{X}$-body axis

$$
T_{j}=k_{t}\left(\omega_{j}\right)^{2}
$$

to get the plane sustentation force. However, the friction between the propeller and the air also causes a parasitical drag moment around the $\mathrm{X}$-body axis

$$
\delta_{j}=k_{d}\left(\omega_{j}\right)^{2}
$$

which hampers the plane controllability. Thus, it is worth investing time to find the best motor-propeller combination. For this work prototype, we have opted for a motor Racestar BR2205, 2300Kv, with a 3-blade propeller 5051, all powered with a 4S LiPo battery. Propellers coefficients $k_{t}$ and $k_{d}$ in Table 1 have been experimentally identified according the procedure in [5].

The propeller rotation axis changes its orientation as the craft rotates. This induces a gyroscopic torque

$$
\mathbf{M}_{\boldsymbol{g}}=\left[\begin{array}{c}
I_{j}^{X} \dot{\omega}_{j} \\
I_{j}^{X} \omega_{j} r \\
-I_{j}^{X} \omega_{j} q
\end{array}\right]
$$

where $I_{j}^{X}$ is the moment of inertia of the rotor around the X-body axe.

Finally, the craft weight in the earth frame responds to

$$
\mathbf{F}_{\boldsymbol{w}}^{\mathbf{E}}=\left[\begin{array}{c}
0 \\
0 \\
-m
\end{array}\right]
$$

where $m$ is the mass of the aircraft and $g$ is the gravity. Then, this force is conveniently rotated to the body frame giving

$$
\mathbf{F}_{\boldsymbol{w}}=\left[\begin{array}{l}
-m g \sin \theta \\
g \sin \phi \cos \theta \\
g \cos \phi \cos \theta
\end{array}\right]
$$

\subsection{Non-linear model}

Substituting external forces and moments (Section 2.2) in generic forces and moments $F_{x}, F_{y}, F_{z}, M_{x}, M_{y}, M_{z}$ in (1) and (2), and rearranging, it yields the dynamic non-linear model of motion in the body frame:

$$
\left\{\begin{array}{c}
-g \sin \theta+\frac{k_{t}\left(w_{j}\right)^{2}}{m}-\frac{F_{a_{X}}}{m}+v r-q w=\dot{u} \\
g \sin \phi \cos \theta+\frac{F_{a_{Y}}}{m}+p w-r u=\dot{v} \\
g \cos \phi \cos \theta-\frac{F_{a_{Z}}}{m}+u q-v p=\dot{w} \\
\frac{I_{j}^{X} \dot{\omega}_{j}}{I_{x x}}+\frac{k_{d}\left(w_{j}\right)^{2}}{I_{x x}}-\frac{\frac{1}{2} \rho V^{2} s c_{l} b}{I_{x x}}-\frac{\left(I_{x x}-I_{z z}\right) q r}{I_{x x}}=\dot{p} \\
\frac{I_{j}^{X} \omega_{j} r}{I_{y y}}+\frac{\frac{1}{2} \rho V^{2} S c_{m} c}{I_{y y}}-\frac{\left(I_{z z}-I_{x x}\right) p r}{I_{y y}}=\dot{q} \\
\frac{-I_{j}^{X} \omega_{j} q}{I_{z z}}-\frac{\frac{1}{2} \rho V^{2} S c_{n} b}{I_{z z}}-\frac{\left(I_{x x}-I_{y y}\right) p q}{I_{z z}}=\dot{r}
\end{array}\right.
$$

The linear velocities $[u, v, w]^{\mathrm{T}}$ can be transferred to the earth frame by multiplying them by matrix (see [5])

$$
\mathbf{R}^{\mathrm{E} \leftarrow \mathrm{B}}=\mathbf{R}_{x}^{\mathrm{E} \leftarrow \mathrm{B}} \cdot \mathbf{R}_{y}^{\mathrm{E} \leftarrow \mathrm{B}} \cdot \mathbf{R}_{z}^{\mathrm{E} \leftarrow \mathrm{B}},
$$

And after integration, it yields absolute position $[x, y$ $z]^{\mathrm{T}}$. Expression (15) uses Euler angles that can be calculated integrating 


$$
\left[\begin{array}{c}
\dot{\phi} \\
\dot{\theta} \\
\dot{\psi}
\end{array}\right]=\left[\begin{array}{ccc}
1 & \sin \phi \tan \theta & \cos \phi \tan \theta \\
0 & \cos \phi & -\sin \phi \\
0 & \sin \phi \sec \theta & \cos \phi \sec \theta
\end{array}\right] \cdot\left[\begin{array}{c}
\dot{p} \\
\dot{q} \\
r
\end{array}\right]
$$

This also yields absolute orientation $[\phi, \theta, \psi]^{\mathrm{T}}$.

\subsection{Linear model: Longitudinal stability}

In order to develop linear control laws, small signal linear models of (14) will be computed. The linearization process is about deriving the equations regarding all variant parameters, evaluating them on a nominal flight condition $\left(V_{a}=31 \mathrm{~m} / \mathrm{s}, \alpha=-0.5^{\circ}, \beta=0^{\circ}\right)$, and multiplying them by the sensitivity. The result of this process is commonly called stability derivatives in the aeronautic field. Longitudinal stability is used for pitch and height control, and lateral stability for roll and yaw control [3] [6].

The longitudinal stability allows us to observe the behaviour of the linear velocity in X-axis $(u)$, the angle of attack $(\alpha)$, the angular velocity in $\mathrm{Y}$-axis $(q)$ -all them computed in the body frame-, and the pitch angle $(\theta)$ in the earth frame, which is approximated by the integration of the aforementioned angular velocity $q$ under the assumption of small roll angles. The symmetric deflection of flaps $\delta_{E}$ is the actuation variable.

Only the strengths in $\mathrm{X}$ and $\mathrm{Z}$ axes, and the moments in $\mathrm{Y}$ axis will be studied, since they are the only ones deemed to intervene in longitudinal stability. With the coefficients obtained following [7], it yields the longitudinal linear model:

$$
\left(\begin{array}{c}
\dot{u} \\
\dot{\alpha} \\
\dot{q} \\
\dot{\theta}
\end{array}\right)=\left(\begin{array}{cccc}
X_{u}+X_{T U} & X_{a} & 0 & -g \\
\frac{Z_{u}}{U_{0}} & \frac{Z_{\alpha}}{U_{0}} & \frac{Z_{q}+U_{0}}{U_{0}} & 0 \\
M_{u}+M_{T U} & M_{\alpha}+M_{T \alpha} & M_{q} & 0 \\
0 & 0 & 1 & 0
\end{array}\right)\left(\begin{array}{c}
u \\
\alpha \\
q \\
\theta
\end{array}\right)+\left(\begin{array}{c}
X_{\delta_{E}} \\
\frac{Z_{\delta_{E}}}{U_{0}} \\
M_{\delta_{E}} \\
0
\end{array}\right) \delta_{E}
$$

In particular, the state equation

$$
\left(\begin{array}{c}
\dot{u} \\
\dot{\alpha} \\
\dot{q} \\
\dot{\theta}
\end{array}\right)=\left(\begin{array}{cccc}
-0.19 & 0 & 0 & -9.17 \\
0 & -20.27 & 0.96 & 0 \\
4.04 & -3861.27 & -9.90 & 0 \\
0 & 0 & 1.00 & 0
\end{array}\right)\left(\begin{array}{l}
\mathrm{u} \\
\alpha \\
\mathrm{q} \\
\theta
\end{array}\right)+\left(\begin{array}{c}
0.51 \\
-11.05 \\
-2985.33 \\
0
\end{array}\right) \delta_{E}
$$

is obtained for the fixed wind prototype in this work. Accordingly, the following input-output transfer functions

$$
\frac{\theta(s)}{\delta_{E}(s)}=\frac{-2985 s^{2}-18820 s-3487}{s^{4}+30.37 s^{3}+3987 s^{2}+787.8 s+658.7}
$$

$$
\frac{\alpha(s)}{\delta_{E}(s)}=\frac{-10.95 s^{3}-2964 s^{2}-569.6 s-334.3}{s^{4}+30.37 s^{3}+3987 s^{2}+787.8 s+658.7}
$$

are of interest in the longitudinal control strategies.

\section{Control strategy}

In flight dynamics, it is all about controlling the magnitude and orientation of the lift vector. Thus, we will have to study those variables whose effect on the vector are significant. We find that the attack angle controls the magnitude and the sweep angle controls the orientation of the lift vector. Consequently, any manoeuvre of winning or losing height would start with a change in the angle of attack, in the same way that a change in the sweep angle has an inherited change in the lateral position.

The desired attack angle is obtained by controlling a desired pitch angle, which finally will intervene in the altitude control. Similarly, a desired sweep angle is obtained by controlling a desired roll angle, which will intervene in the yaw control.

In this work, two cascaded loops will allow controlling first the pitch angle. Then, considering the attack angle, it will allow controlling the height inside another outer loop.

\subsection{Pitch control architecture}

Figure 4 depicts the pitch control architecture. Block $a(s)$ represents the actuator dynamic, which is here discarded $(a(s)=1)$ in comparison with the rigid solid dynamics $\theta(s) / \delta_{E}(s)$. The pure derivative in the inner loop is actually a mathematical resource, since $q$ is the measurable variable in practice. Thus, gain $K_{q}$ is the controller in the feedback path of the inner loop. The outer loop provides the feedback controller $C \theta(s)$ in the direct path. The control design process is performed from the inner to the outer loop, as it is following detailed.

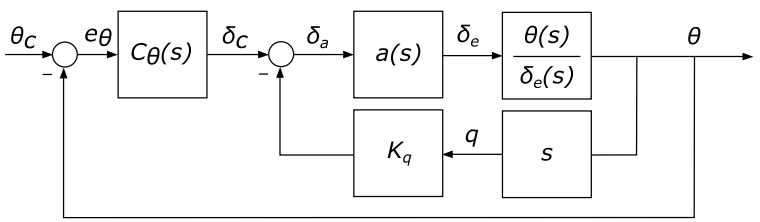

Figure 4: Pitch control architecture

From a pure mathematical point of view the diferenciator in the inner loop mitigates the underdamping (0.244) of dominant poles in $\theta(s) / \delta_{E}(s)$ of (19). Figure 5 depicts this effect in the frequency domain response of $\theta / \delta_{E}$. Then, $K_{q}$ is tuned to achieve a suitable control bandwidth $\omega_{B W}$; acceptable values are between 1 and $10 \mathrm{rad} / \mathrm{s}$. Finally, a value of 


$$
K_{q}=-0.25
$$

achieves a $\omega_{B W}=1.71 \mathrm{rad} / \mathrm{s}$, as Figure 6 shows. Let us remark that a negative control gain is necessary in the inner loop since $\theta(s) / \delta_{E}$ has inverse gain.
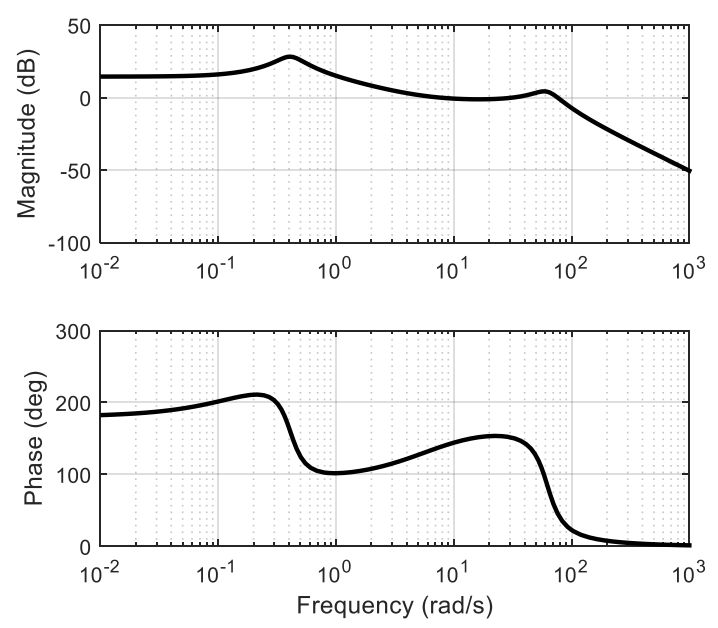

Figure 5: Open-loop frequency response $\theta / \delta_{E}$
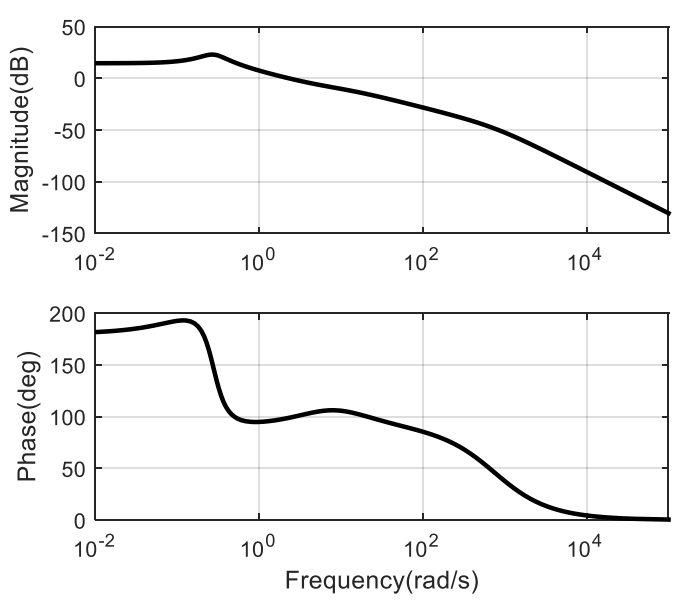

Figure 6: Closed loop frequency response $\theta / \delta_{c}$

Regarding the outer loop design, a proportionalintegral (PI) controller is attempted: first, an integrator to remove the position error and later on a zero to mitigate the integrator effect over medium frequencies guaranteeing enough phase margin (PM) -higher than $40^{\circ}$-. The PI controller gain modulates the gain cross over frequency $\omega_{g c}$ (values between 1 and $10 \mathrm{rad} / \mathrm{s}$ are acceptable). Negative control gain is necessary since $\theta / \delta_{c}$ has inverse gain as phase plot reveals in Figure 6. The final controller at the outer loop is

$$
C_{\theta}(s)=-0.632 \frac{(1+5 s)}{s}
$$

which achieves a PM of $90^{\circ}$ at $\omega_{g c}$ of $9.78 \mathrm{rad} / \mathrm{s}$, as Figure 7 depicts. Finally, the closed-loop frequency response $\theta / \theta_{c}$ reaches $-3 \mathrm{~dB}$ above $\omega_{B W}=5.5 \mathrm{rad} / \mathrm{s}$.
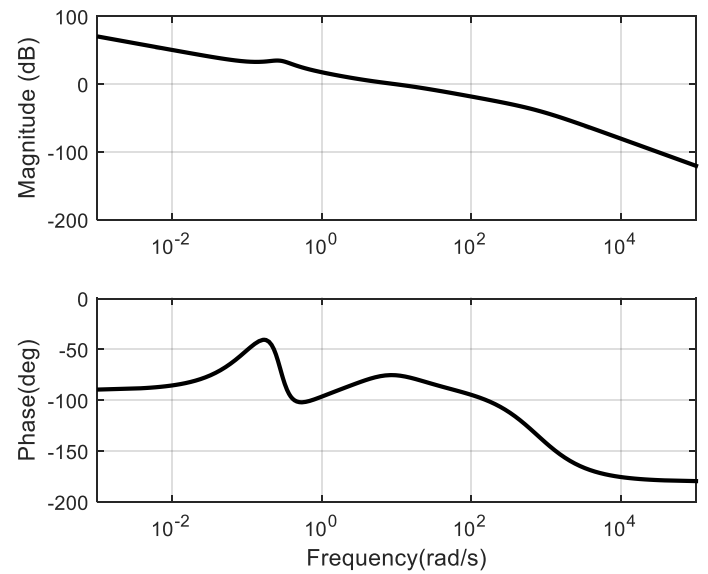

Figure 7: Open-loop frequency response of $\theta / e_{\theta}$

\subsection{Altitude control architecture}

The altitude control consists of another feedback control loop above the pitch control structure $\theta / \theta_{c}$ of Figure 4 , as Figure 8 shows. $C_{h}(s)$ is the feedback controller to be designed. The path angle

$$
\gamma(t)=\theta(t)-\alpha(t)
$$

is related to the altitude such that

$$
\dot{h}=U_{0} \sin \gamma \approx U_{0} \gamma
$$

where $U_{0}$ is the craft velocity that is equal to $V_{a}=31$ $\mathrm{m} / \mathrm{s}$ when $\alpha=\beta=0^{\circ}$.

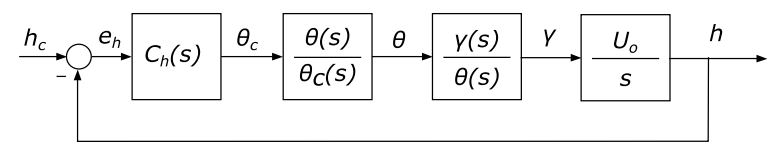

Figure 8: Altitude control architecture

Considering (23) (19) and (20), it is obtained

$$
\frac{\gamma(\mathrm{s})}{\theta(\mathrm{s})}=\frac{-0.003667(\mathrm{~s}+39.77)(\mathrm{s}-41.91)(\mathrm{s}+0.1727)}{(\mathrm{s}+6.115)(\mathrm{s}+0.191)}
$$

which can be approximated by

$$
\frac{\gamma(\mathrm{s})}{\theta(\mathrm{s})} \approx \frac{5.4189}{s+6.115}
$$

in order to simplify the design process. Finally, $h / \theta_{c}$ presents the frequency response in Figure 9. 
A PI controller cannot achieve acceptable PM (above $45^{\circ}$ ) for good stability and high enough cross overfrequencies (1-10 rad/s) for a good performance. However, the final controller

$$
C_{h}(s)=\frac{0.05(1+s / 0.9)(s+1)}{s(1+s / 2.4)}
$$

achieves a $\mathrm{PM}$ of $51.45^{\circ}$ and $\omega_{c g}=2.54 \mathrm{rad} / \mathrm{s}$ as Figure 10 shows. The closed-loop bandwidth of $h / h_{c}$ is $\omega_{B W}=4.25 \mathrm{rad} / \mathrm{s}$.
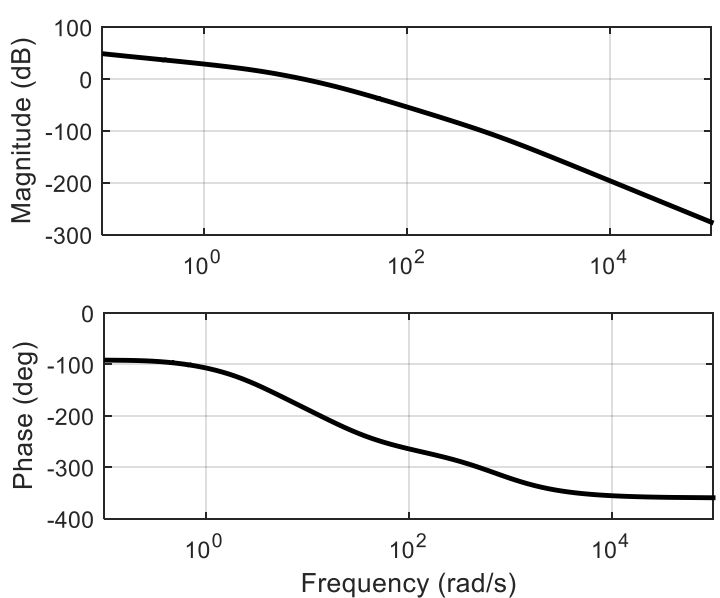

Figure 9: Frequency response of $h / \theta_{c}$
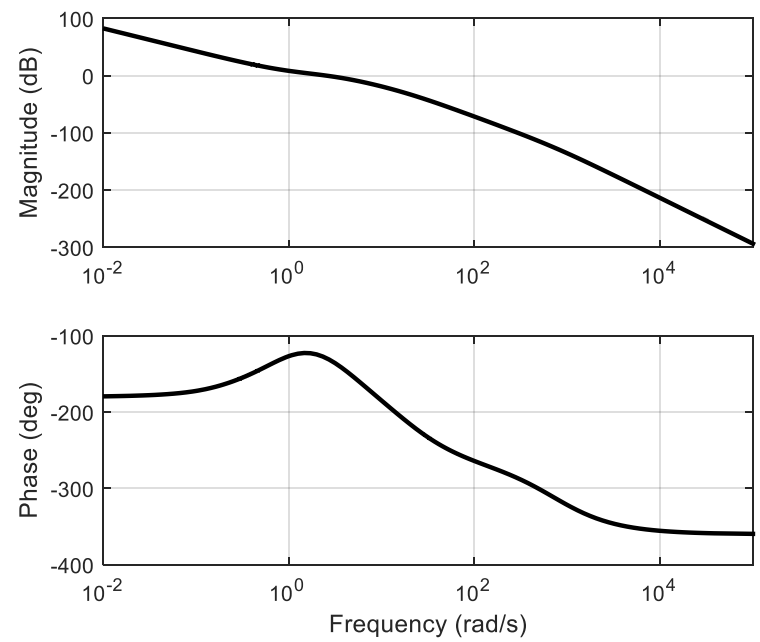

Figure 10: Open-loop frequency response of $h / e_{h}$

\subsection{Validation in the non-linear model}

Longitudinal control is being tested in the fully coupled system with all the non-linear behaviours. The non-linear model in Section 2.3 has been implemented in a "User-Defined Block" in Simulink with the symmetric and asymmetric flap deflection as control inputs, and the three Euler Angles and altitude as controlled outputs. Using this block, the aforementioned control loops (Sections 3.1 and 3.2) have been also implemented in the script. Besides, yaw must be controlled to zero, using a similar control structure (Figure 11) as in the height control, let us note as it includes inner roll control loops, similar to the pitch control architecture.

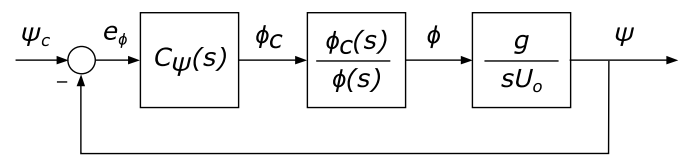

Figure 11: Yaw control architecture

Figure 12 depicts several time responses related to height reference changes of step and ramp type. Plot (a) depicts the height tracking response (black) to reference signals (grey) of different nature; plot (b) shows the pitch that is demanded (grey) and how it is attained (black) by the inner loop; and plot (c) shows the deflection angle variation.
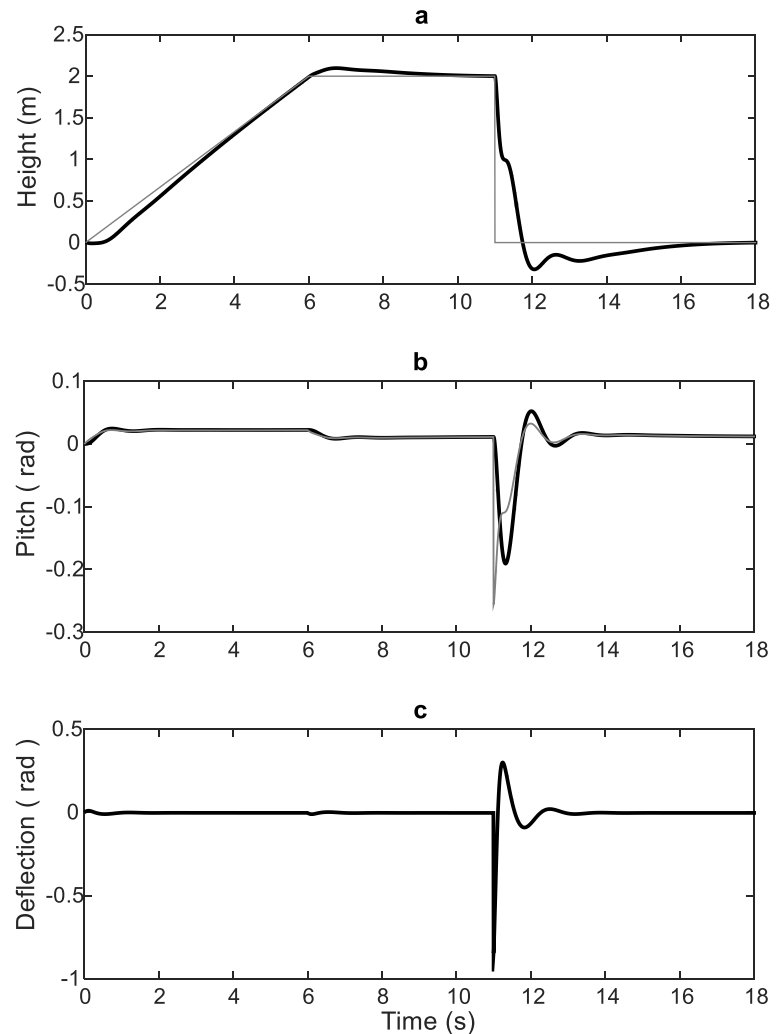

Figure 12: Altitude control performance

\section{Conclusions}

In this article, we have presented the mathematical model of a fixed wing aircraft. For a hand-made protype, we have identified aerodynamic and physical parameters such as aerodynamic coefficients, inertias or weights, among others, mainly using the opensource software XFLR-5. Furthermore, we have 
isolated all the moments and strengths in the system: weight, aerodynamic forces and moments, thrust, drag sweep.

Following Newton-Euler formulation, we have come up with a non-linear model, which has been linearized in order to apply linear control theory.

A longitudinal stability model has been used to design feedback control loops of a cascade structure. Frequency domain techniques were used to design PID type controllers. An inner feedback loop controlled the pitch angle by conveniently acting on the flap deflection. Then, an outer loop allowed tracking the desired altitude.

Achieving this controlled model is the start of a way for improvement and allows us to contribute to the creation of navigation systems, laying the foundations of new work lines.

The development of the model and its control is the first step to design optimized control strategies and to explore new possibilities in the field.

\section{Acknowledgements}

The authors gratefully appreciate the support given by La Rioja Government under grant ADER 2017-I-IDD00035 and the support given by the University of La Rioja under grant REGI 2018/42.

\section{References}

[1] Mark Edward Peterson. (2006) "The UAV and the current and future regulatory construct for integration into the national airspace system". Journal of Air Law and Commerce, vol. 71(5) pp. 521-612.

[2] Alexander V. Koldaev. (2007) "Non-military UAV applications".

[3] David K. Schmidt. (2012) "Modern Flight Dynamics”, McGraw-Hill International Edition.

[4] Guidelines for XFLR5 V0.03. (2009) "XFLR5 Analysis of foils and wings operating at low Reynolds numbers".

[5] R.Rico, P. Maisterra, M. Gil-Martínez, J. RicoAzagra, S. Nájera (2015). "Identificación experimental de los parámetros de un cuatrirrotor". In XXXVI Jornadas de Automática.

[6] Smetana, Frederick O. Delbert C. Summery and W. Donlad Johnson (1972), "Riding and Handling Qualities of light Aircraft-A Review and Analysis". National aeronautics and space administration. Washington D.C

[7] Esteban. S., (2001) "Static and dynamic analysis of an unconventional plane: Flying wing”. In AIAA Atmospheric Flight Mechanics Conference and Exhibit.

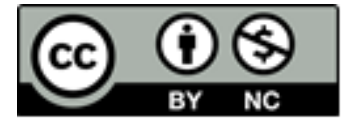

(C) 2018 by the authors. Submitted for possible open access publication under the terms and conditions of the Creative Commons Attribution CC-BY-NC 3.0 license (https://creativecommons.org/licenses/by-nc/3.0). 